\title{
Test Gas Generation from Pure Liquids: An Application-Oriented Overview of Methods in a Nutshell
}

\author{
Yue Li, ${ }^{1}$ Tim Täffner, ${ }^{2}$ Michael Bischoff, ${ }^{3}$ and Bernd Niemeyer ${ }^{2}$ \\ ${ }^{1}$ Technical University Munich, Arcisstraße 21, 80333 Munich, Germany \\ ${ }^{2}$ Helmut-Schmidt-University/University of the Federal Armed Forces Hamburg, Holstenhofweg 85, 22043 Hamburg, Germany \\ ${ }^{3}$ Lübeck University of Applied Sciences, Mönkhofer Weg 239, 23562 Lübeck, Germany \\ Correspondence should be addressed to Bernd Niemeyer, niemeyer@hsu.-hh.de
}

Received 6 September 2011; Revised 18 December 2011; Accepted 18 December 2011

Academic Editor: D. Yu. Murzin

Copyright (C) 2012 Yue Li et al. This is an open access article distributed under the Creative Commons Attribution License, which permits unrestricted use, distribution, and reproduction in any medium, provided the original work is properly cited.

The generation of test gas from pure liquids has a wide variety of applications in laboratory and field experiments, for which the quality of the test gas is of significance. Therefore, various methods for test gas generation have been designed. Each method has unique advantages and disadvantages. Thus, a short overview is presented within the scope of this paper. Furthermore, a common bubbler system is presented, which was built to generate test gas from volatile organic compounds for experimental usage in laboratory applications. An analysis is conducted with respect to the generated concentrations at different temperatures and flow rates of the diluting gas. Accuracy and stability of this method are investigated.

\section{Introduction}

The generation of test gas from pure liquids, for example, volatile organic compounds (VOCs), is of great importance for laboratory and field experiments. There are several major applications for it:

(i) Adsorption analysis of isotherms, breakthrough, and kinetics.

(ii) Standard gas for calibration of analytical instruments, for example, gas chromatography (GC).

(iii) Safety system verification/calibration.

(iv) Calibration of air quality analysis and pollution control systems.

(v) Chemical vapour deposition (CVD).

Various methods have been developed for the generation of test gas. They can be divided into two categories: static and dynamic methods [1-4].

The injection of liquid and its evaporation in an enclosed container with defined volume of diluting gas constitute the functional principles of all static methods. The metal, glass, or plastic container could be employed without any other complex apparatus, which is simple and inexpensive.
However, one of the crucial drawbacks of these methods is the appearance of adsorption and condensation on the wall of the container [2]. Therefore, the concentration of the test gas cannot be reliable at higher concentrations. Another drawback is that only a limited amount of test gas is generated once. Moreover, leakages and pressure changes exert an effect.

Compared with static methods, dynamic methods are based on a continuous diluting gas flow through the generation system and the mixture with the vapour at a known generation rate [2]. Though these methods are more complex and expensive, but they show important advantages, such as a negligible effect of the adsorption and condensation at the state of equilibrium, continuous test gas generation without volume limit, continuous dilution providing a wide concentration range, and possible control of test gas condition for temperature, relative humidity, and flow rate [3].

\section{Comparison of Dynamic Methods}

Hence, dynamic methods have a wide variety of applications. With respect to the methods of generation and discharge of the vapour into the diluting gas, dynamic methods can be 
further classified as injection methods, permeation methods, diffusion methods, and evaporation methods $[2,4]$.

2.1. Injection Methods. Injection Methods are employed to prepare test gas by injecting liquid into the diluting gas. Different injection devices, for example, syringes, motor-driven syringes, and syringe pumps have been widely applied. The advantages of the injection with a syringe are that a test gas with a well-known concentration can be prepared, and variable concentrations may be obtained applying different flow rates of the diluting gas [5]. In order to achieve total volatilization of the liquid, injected into the diluting gas, atomizers and heaters of the mixing chamber are frequently applied. Meanwhile, the heating system will prevent the vapour from condensation. However, significant variation of concentrations in the mixing chamber could appear after injection, thus an expanded mixing chamber is required to balance the fluctuation [6]. Another shortcoming of the injection method is associated with the limited volume of the syringe and its need to refill [3].

2.2. Permeation Methods. Permeation Methods are based on Fick's law. Vapour generated from a liquid diffuses through a membrane. Among various devices, the permeation tube is most commonly utilized. A permeation tube is prepared by sealing a liquid in a tube made of polymer materials. The permeation rate reaches a constant value if it is kept at a constant temperature. The sealed tube acts as a stable source generating the vapour, which then permeates into the diluting gas flow. Parameters, for example, the operating temperature, the tube length and its material, and the wall thickness influence the permeation rate, which will further affect the concentration of the relevant substance in the carrier gas. Temperature is the most significant parameter for the permeation methods. It has been described that the permeation rate varies by $10 \%$ with a change in temperature of $1 \mathrm{~K}[2]$. Thus, the permeation methods require precise temperature control to ensure the accuracy of the gas concentration. Different concentrations are also available by adjusting the flow rate of the diluting carrier gas. Besides the permeation tube, complete apparatus for the preparation of test gas using permeation technique is commercially available, which is known as "calibrator" [2]. The permeation methods are available for many components, including condensable gases, permanent gases, liquids, sublimating solids, and many semivolatile compounds. Permeation methods are considered as an effective and accurate way for preparing low concentration test gas, at $\mathrm{ppm}(\mathrm{v} / \mathrm{v})$ to $\mathrm{ppb}(\mathrm{v} / \mathrm{v})$ levels [7]. However, the disadvantages of these methods are a long initial time for reaching permeation equilibrium, relative high costs, and continuous emission from the source without shutting up possibility $[2,4]$.

2.3. Diffusion Methods. Diffusion Methods rely on the principle of diffusion of the vapour of a liquid from a container through capillaries or directly from a capillary and subsequent mixing with a diluting gas flow. The diffusion conditions, that is, temperature, pressure, concentration gradient, length and diameter of the capillary, and the flow rate of the diluting gas $[3,8,9]$, must be maintained to obtain a constant concentration of test gas. Similar to the permeation methods, the temperature has a key effect: A variation in temperature of $1 \mathrm{~K}$ at a room temperature level changes the diffusion rate by approx. 5\% [10]. The diffusion methods provide vapour concentrations between 0.1 and $100 \mathrm{ppm}(\mathrm{v} / \mathrm{v})$ [11]. They reveal similar advantages and disadvantages as the permeation methods, have a wide applicability, and are suitable for many compounds. However, it is impossible for the preparation of a multicomponent mixture in one diffusion vessel, unless several vessels filled with pure components are applied [12]. Furthermore, they also need long period to obtain diffusion equilibrium for steady gas generation.

2.4. Evaporation Methods. Evaporation Methods represent the simplest functional principle. The diluting carrier gas is utilized to bubble through a liquid or pass over a liquid surface. In the literature $[2,13]$, it is suggested that the flow rate of the diluting gas should be sufficient low in order to ensure that the gas concentration reaches the state of saturation. The steam of the saturated vapour could be subsequently diluted with an additional gas flow or may be recooled using a heat exchanger in order to reduce the concentration and stabilize on a lower level. Evaporation methods are inexpensive and have a short stabilization period for the test gas generation, compared to permeation and diffusion methods. Furthermore, these methods are often exerted to humidify air.

In Table 1, the accuracy of these four different dynamic methods is presented.

Based on these four most common dynamic methods discussed above, a variety of novel methods has been designed for the purpose of test gas generation, for example, microwave-assisted methods [20], thermal decomposition of immobile compounds [21], and the commercial Controlled Evaporation and Mixing (CEM) liquid delivery system [22].

The CEM system controls the required quantity of liquid to reach the desired concentration under room temperature with a liquid mass flow meter. The defined quantity of liquid is subsequently mixed with the carrier gas, and the mixture is totally evaporated within the evaporator. Therefore, any concentration could be generated by adjusting the flow rates of liquid and carrier gas, respectively. This method provides a very stable and accurate vapour flow, meanwhile the response period is shorter than those of conventional methods. Another advantage of this method is the instantaneous generation of vapour of a mixture of liquids with different vapour pressures, which is hard to achieve with conventional methods [22].

\section{Experimental}

In order to analyze and evaluate a common and popular dynamic method, a test gas generation system based on an Impinger bubbler was created and characterized. This system substantiates an application-oriented method to provide test gas with a specific VOC concentration using common laboratory equipment. The experimental part aims at the 
TABLE 1: Accuracy of test gas generated based on different methods.

\begin{tabular}{lcl}
\hline Technique & Accuracy & Remarks \\
\hline Injection & $\begin{array}{c}5-9 \%(\mathrm{v} / \mathrm{v}) \\
{[14,15]}\end{array}$ & $\begin{array}{l}\text { (i) The accuracy strongly depends on the accuracy of the injection devices. } \\
\text { (ii) When multicomponents test gas is generated, the accuracy is not as precise as for pure components. }\end{array}$ \\
\hline $\begin{array}{l}\text { Permeation } \\
2-5 \%(\mathrm{v} / \mathrm{v}) \\
{[7,16-18]}\end{array}$ & $\begin{array}{l}\text { (i) The main source of the uncertainty comes from the permeation rate, which is influenced by } \\
\text { thermodynamic and physical state variables, such as temperature, pressure and gas flow. } \\
\text { (ii) Permeation methods can be used to generate very low concentrations (ppb level), the uncertainty of } \\
\text { the calibration itself also has a strong influence on the overall accuracy. }\end{array}$ \\
$\begin{array}{l}\text { Diffusion } \\
3-5 \%(\mathrm{v} / \mathrm{v})\end{array}$ & $\begin{array}{l}\text { (i) The most important uncertainty is the diffusion rate. The variation of it is related to the compound } \\
\text { itself, the design of the diffusion device, and thermodynamic state variables. } \\
\text { (ii) In analogy to to the permeation method, the calibration itself contributes to the overall uncertainty. }\end{array}$ \\
$\begin{array}{l}\text { Evaporation } \\
5-15 \%(\mathrm{v} / \mathrm{v})\end{array}$ & $\begin{array}{l}\text { (i) The marked deviation results from the dilution system and temperature variation. } \\
\text { (ii) The vapour pressure of the compound is also one considerable factor. The error can be minimized for } \\
\text { compounds with a low vapour pressure. }\end{array}$ \\
\hline
\end{tabular}

discovery and determination of factors which limit the accuracy. Thus, two VOCs with much differing polarity, acetone and $\mathrm{n}$-hexane, were utilized to generate the test gas. Both were purchased from Sigma-Aldrich (Seelze, Germany) and were of analytical purity grade.

3.1. Construction and Operation of the Bubbler System. The design of the bubbler system employed in this study is presented in Figure 1. The compressed air is introduced into the system and passes through the purifier in order to remove the disturbing components, for example, water vapour, volatile organic compounds, and particles, from the compressed air. The flow regulator sets a constant inlet pressure of 3 bars, providing a steady flow. An EL-FLOW series Mass Flow Meter and Controller (Bronkhorst Mättig, Kamen, Germany) is used to regulate the flow rate of the compressed air through the system. Later, the flowcontrolled air passes through the Impinger gas scrubber filled with pure VOC liquid, therefore the test gas is produced applying the bubbling process. The temperature of the Impinger gas scrubber is controlled by a thermostat circulating water. The accuracy of the thermostat is $\pm 0.02 \mathrm{~K}$. All parts of the system are connected with PTFE tubings, which are inert and minimize the loss of compounds due to adsorption onto the walls.

In order to enlarge the contact surface, glass wool is immerged into the cavity of the Impinger gas scrubber. At the outlet glass wool is used to prevent liquid droplets leaving the system.

3.2. Sample Collection. The generated test gas is sampled in Tedlar bags at the outlet. They have a polypropylene fitting, which contains a syringe port with Teflon lined septum, a hose connection, and a shut-off valve for the hose connection. Before use, the bags are thoroughly flushed with purified air and then vacuumed with a vacuum pump for at least three times, and the absence of residues is determined with a blank test. The volume of the Tedlar bags is $10 \mathrm{~L}$. The sampling period was chosen with respect to the flow rate.

3.3. GC/FID Analyses. For analyses of the test gas, the gas chromatograph Clarus 500 GC combined with a Flame Ionization Detector (FID) (both from Perkin Elmer LAS,
TABLE 2: Saturated gas concentration of acetone and n-hexane.

\begin{tabular}{llc}
\hline \multirow{2}{*}{ Compounds } & \multicolumn{2}{c}{ Saturated concentration $(\mathrm{mmol} / \mathrm{L})$} \\
& $5^{\circ} \mathrm{C}$ & $20^{\circ} \mathrm{C}$ \\
\hline Acetone & 5.25 & 10.1 \\
n-Hexane & 3.41 & 6.15 \\
\hline
\end{tabular}

Rodgau-Jügesheim, Germany) and a high performance capillary-column (Macherey-Nagel, Düren, Germany) was used. High-purified helium (5.0) is applied as carrier gas for the sample through the capillary column. Before the GC analysis, a calibration of the GC with respect to the two types of VOC and their concentration ranges used in this study was conducted.

\section{Results and Discussion}

The bubbler system is based on the evaporation of VOC to generate test gas. The saturated vapour pressure of the compound could be obtained from the literature applying the Antoine equation [23].

Furthermore, the saturation concentration of the compound in the gaseous mixture was calculated based on the ideal gas law. In Table 2, the calculated theoretical concentrations are listed. All of these values are valid under standard atmospheric pressure of 1013 mbar.

4.1. Comparison of Measured and Theoretical Test Gas Concentrations. In order to evaluate the above-described Impinger gas scrubber system, two influencing factors, the evaporation temperature and the flow rate, are analyzed for acetone and n-hexane, respectively. For every set point at least ten measurements were performed and statistically evaluated.

After analyses with the GC/FID system, the results are presented in Figures 2 and 3. A pair of samples is taken and analyzed. The mean values and the deviations are also presented in these figures. Figure 2 shows the measured results for acetone. It can be seen that the concentrations for the lower evaporation temperature of $5^{\circ} \mathrm{C}$ differ significantly from the saturation concentrations presented in Table 2. In 


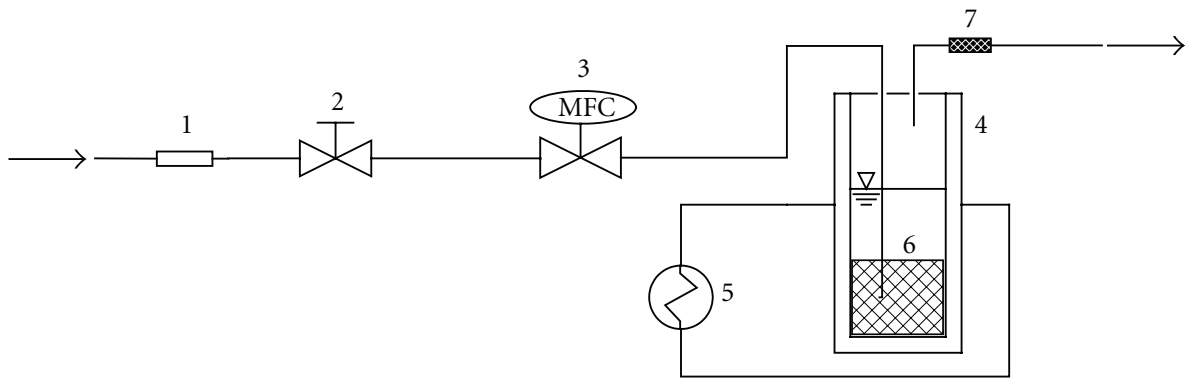

FIGURE 1: Bubbler system for the generation of test gas: (1) purifier with adsorbent, (2) flow regulator, (3) mass flow controller, (4) Impinger gas scrubber, (5) thermostat, and (6), (7) glass wool.

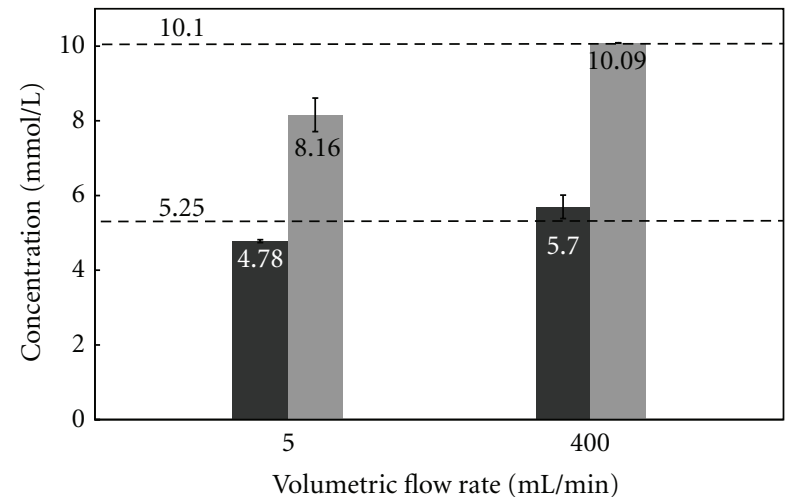

- $5^{\circ} \mathrm{C}$

- $20^{\circ} \mathrm{C}$

FIGURE 2: Acetone test gas concentration sampled from the bubbler system and calculated concentrations from Table 2.

this case, the differences are $\pm 10 \%$, while the measured values for an evaporation temperature of $20^{\circ} \mathrm{C}$ and a volume flow of $400 \mathrm{~mL} / \mathrm{min}$ are in very good agreement.

The lower volume flow of $5 \mathrm{~mL} / \mathrm{min}$ results in both cases in less accurate concentrations.

The measured n-hexane concentrations are presented in Figure 3. The standard deviations of these four experimental set points are significantly higher. At the evaporation temperature of $5^{\circ} \mathrm{C}$, differences to the saturation value from Table 2 of $\pm 3 \%$ are determined. At a higher temperature of $20^{\circ} \mathrm{C}$, the measured concentration reaches only $70 \%$ of the saturation value.

Within recent literature sources [2,13], the assumption is given that the test gas concentration is closer to the saturation when the bubbler system works with a low flow rate, as the gas contact time within the liquid is longer. However, the experimental results do not obey this theoretical prediction. For both compounds, it is clearly shown that higher flow rates of the diluting carrier gas lead to higher concentrations of generated test gas. The concentration of acetone test gas is almost saturated at high flow rates, whereas it is not the case at low flow rates. With higher flow rates, the concentration of acetone gas is increased by about $19 \%$ raising the flow rate from 5 to $400 \mathrm{~mL} / \mathrm{min}$ at $5^{\circ} \mathrm{C}$ and $24 \%$ at $20^{\circ} \mathrm{C}$. The $\mathrm{n}$ hexane gas concentration definitely increases with increasing

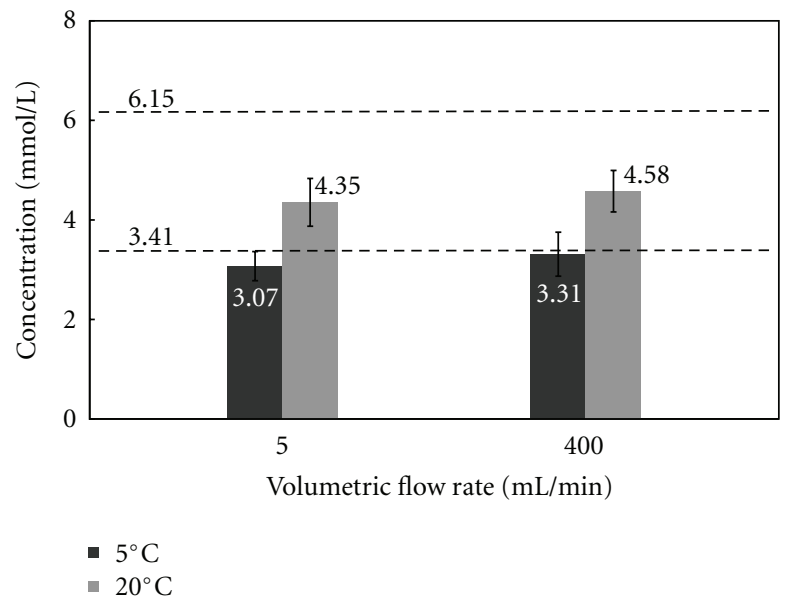

Figure 3: n-Hexane test gas concentration sampled from the bubbler system system calculated concentrations from Table 2.

flow rate by about $8 \%$ at $5{ }^{\circ} \mathrm{C}$ and $5 \%$ at $20^{\circ} \mathrm{C}$. The influence of the flow rate according to $n$-hexane is smaller than that of acetone. A possible reason to explain this could be the vapour pressure of n-hexane, which is smaller than the vapour pressure of acetone at the same temperature. Thus $\mathrm{n}$-hexane is less volatile than acetone. Consequently, the flow rate has a bigger influence on the more volatile compound. A possible reason for this unexpected behavior is also seen in the way of bubbling at the end of the immersion tube. At low volume flow rates, the created bubbles are bigger, and raise slowly through the liquid, driven by buoyancy. Thus the laminar movement of large bubbles does not result in perturbation and mixing and furthermore the state of apparently saturation cannot be reached completely.

4.2. Accuracy and Stability of the Bubbler System. Based on the results in Figures 2 and 3, the bubbler system shows significant fluctuations in test gas generation, with which the deviation of the VOC concentration varies from $\pm 0.1 \%$ to $\pm 13 \%$. This is obviously not ideal, compared to the more stable generated concentrations by permeation and diffusion methods. The variations of the temperature and the flow rate of the diluting gas also contribute to it. Nevertheless, the determination of preferable set points, for example, nhexane at $5^{\circ} \mathrm{C}$ and flow rates between 5 and $400 \mathrm{~mL} / \mathrm{min}$ 
or acetone at $400 \mathrm{~mL} / \mathrm{min}$ and temperatures between 5 and $20^{\circ} \mathrm{C}$ are usable for a broad range of concentrations. Applying the above-described preferable set-points, small standard deviations of $\pm 0.1 \%$ resulting in a high stability of the experimental process and a good convergence between the calculated and measured concentration of a few $\%$ can be reached.

The generated test gas concentration for applying the evaporation method is strongly depending on the evaporation rate of the compound. Thus the depth of the column of liquid above the dispersion tube [4] can also be an influencing factor for the rate of evaporation. As this depth changes slightly during the evaporation process, the residence time of the gas varies and thus the degree of saturation may shift. Other factors, for example, cooling of the liquid due to evaporation process and pressure change, also affect the evaporation rate. During the runtime of the experiments presented above, a change of the liquid level within the gas scrubber was not measureable. Temperature differences and heat fluxes during the startup phase were avoided by conducting the measurements when the system reaches stable conditions.

\section{Conclusions}

The presented methods constitute a broad variety of experimental set ups with substantial differences in accuracy and effort, which can be made to optimize the procedures. The experimentally analyzed bubbler system based on the evaporation method is a convenient and inexpensive approach for the generation of test gas. Advantageous is the possibility to generate the test gas continuously with respect to the volume of the gas scrubber. However, the generated concentration depends on several factors, which must be carefully controlled and adjusted in order to ensure the accuracy of the demanded test gas concentration. After all it can be stated that the generated test gas must be analyzed with analytical instruments in order to ensure its quality before use within the scope of laboratory and field experiments. This widespread employed technique gives reliable and accurate results only if the mentioned influencing parameters are controlled precisely. Additionally the validation of the generated gas with analytical instruments (e.g. GC/FID) is recommended for control, calibration, and further adjustment of the desired test gas concentration by dilution with clean air.

\section{Acknowledgments}

This work was achieved in the scope of the project "SAFE: Semipermeable Anzüge für Einsatzkräfte," which is funded by the German Federal Ministry of Education and Research (BMBF) in context of the program "Research for Civil Protection" under support code 13N9806 as well as the project "CATE-B: China Applied Technologies for EnvironmentBiogas," which is funded by the Robert Bosch Stiftung; Sustainable Partners-Partners for Sustainability Sincere thanks are given to all contributors.

\section{References}

[1] P. Konieczka, J. Namiesnik, and J. F. Biernat, "Generation of standard gaseous mixtures by thermal decomposition of surface compounds. standard mixtures of thiols," Journal of Chromatography, vol. 540, no. 1-2, pp. 449-455, 1991.

[2] J. Namieśnik, "Generation of standard gaseous mixtures," Journal of Chromatography A, vol. 300, pp. 79-108, 1984.

[3] J. A. Koziel, P. A. Martos, and J. Pawliszyn, "System for the generation of standard gas mixtures of volatile and semivolatile organic compounds for calibrations of solid-phase microextraction and other sampling devices," Journal of Chromatography A, vol. 1025, no. 1, pp. 3-9, 2004.

[4] R. S. Barratt, "The preparation of standard gas mixtures: a review," Analyst, vol. 106, no. 1265, pp. 817-849, 1981.

[5] G. O. Nelson and K. S. Griggs, "Precision dynamic method for producing known concentrations of gas and solvent vapor in air," Review of Scientific Instruments, vol. 39, no. 6, pp. 927928, 1968.

[6] G. O. Nelson, Controlled Test Atmospheres, Principles and Techniques, Ann Arbor Science Publishers, Ann Arbor, MI, USA, 1971.

[7] S. Tumbiolo, L. Vincent, J. F. Gal, and P. C. Maria, “Thermogravimetric calibration of permeation tubes used for the preparation of gas standards for air pollution analysis," Analyst, vol. 130, no. 10, pp. 1369-1374, 2005.

[8] J. P. Spinhirne and J. A. Koziel, "Generation and calibration of standard gas mixtures for volatile fatty acids using permeation tubes and solid-phase microextraction," American Society of Agricultural Engineers, vol. 46, no. 6, pp. 1639-1646, 2003.

[9] D. Helmig, T. Revermann, J. Pollmann et al., "Calibration system and analytical considerations for quantitative sesquiterpene measurements in air," Journal of Chromatography A, vol. 1002, no. 1-2, pp. 193-211, 2003.

[10] G. O. Nelson, Gas Mixtures: Preparation and Control, CRC Press, Boca Raton, FL, USA, 1992.

[11] J. M. McKelvey and H. E. Hoelscher, "Apparatus for preparation of very dilute gas mixtures," Analytical Chemistry, vol. 29, no. 1, p. $123,1957$.

[12] A. Naganowska-Nowak, P. Konieczka, A. Przyjazny, and J. Namieśnik, "Development of techniques of generation of gaseous standard mixtures," Critical Reviews in Analytical Chemistry, vol. 35, no. 1, pp. 31-55, 2005.

[13] E. E. Campbell, M. F. Milligan, and H. M. Miller, "Evaluation of methods for the determination of halogenated hydrocarbons in air," American Industrial Hygiene Association Journal, vol. 20, no. 2, pp. 138-141, 1959.

[14] H. Hori and Y. Yanagisawa, "A new vapor generator of multicomponent organic solvents using capillary effect," Environmental Science and Technology, vol. 27, no. 10, pp. 2023-2030, 1993.

[15] D. J. Troy, "Measurement of atmospheric pollution by ultraviolet photometry," Analytical Chemistry, vol. 27, no. 8, pp. 1217-1221, 1955.

[16] P. P. Ballesta, A. Baldan, and J. Cancelinha, "Atmosphere generation system for the preparation of ambient air volatile organic compound standard mixtures," Analytical Chemistry, vol. 71, no. 11, pp. 2241-2245, 1999.

[17] D. Knopf, "Continuous dynamic-gravimetric preparation of calibration gas mixtures for air pollution measurements," Accreditation and Quality Assurance, vol. 6, no. 3, pp. 113-119, 2001.

[18] P. C. Maria, J. F. Gal, M. Balza, E. Peré-Trepat, and S. Tumbiolo, "Using thermogravimetry for weight loss monitoring of 
permeation tubes used for generation of trace concentration gas standards," Analytical Chemistry, vol. 74, no. 1, pp. 305307, 2002.

[19] M. Gautrois and R. Koppmann, "Diffusion technique for the production of gas standards for atmospheric measurements," Journal of Chromatography A, vol. 848, no. 1-2, pp. 239-249, 1999.

[20] G. Xiong and J. Pawliszyn, "Microwave-assisted generation of standard gas mixtures," Analytical Chemistry, vol. 74, no. 10, pp. 2446-2449, 2002.

[21] E. Przyk, P. Konieczka, J. Szczygelska-Tao, R. Teschner, J. F. Biernat, and J. Namieśnik, "Use of porous glass and silica gel as support media of a surface compound for generation of analytes in gaseous standard mixtures. A new method for the determination of the amount of analyte generated," Journal of Chromatography A, vol. 928, no. 1, pp. 99-108, 2001.

[22] CEM Liquid Delivery System, "Bronkhorst High-Tech B.V.," http://www.bronkhorst.com/files/downloads/brochures/cem .pdf.

[23] R. H. Perry, D. W. Green, and J. O. Maloney, Perry's Chemical Engineers Handbook, McGraw-Hill, New York, NY, USA, 6th edition, 1984. 

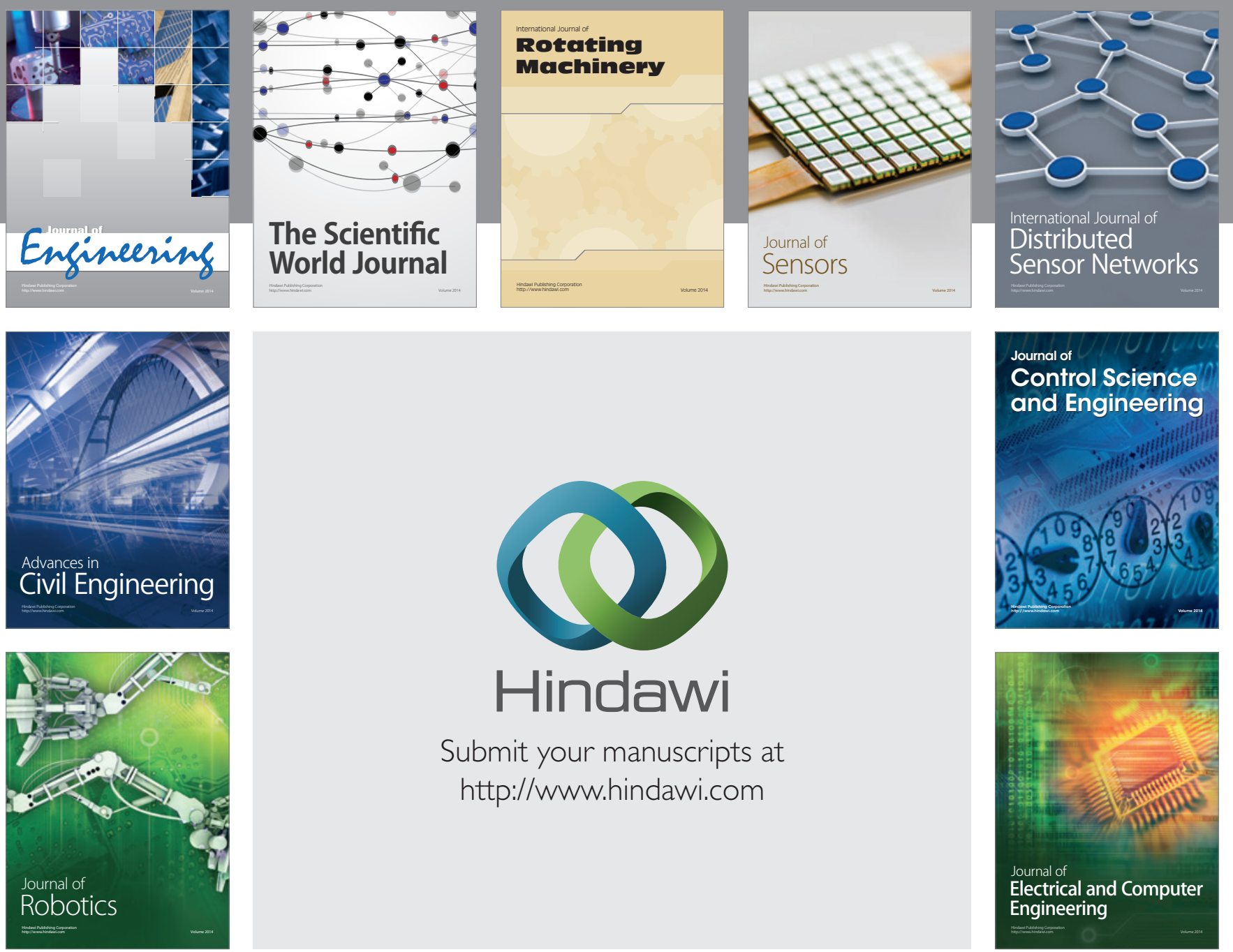

Submit your manuscripts at

http://www.hindawi.com
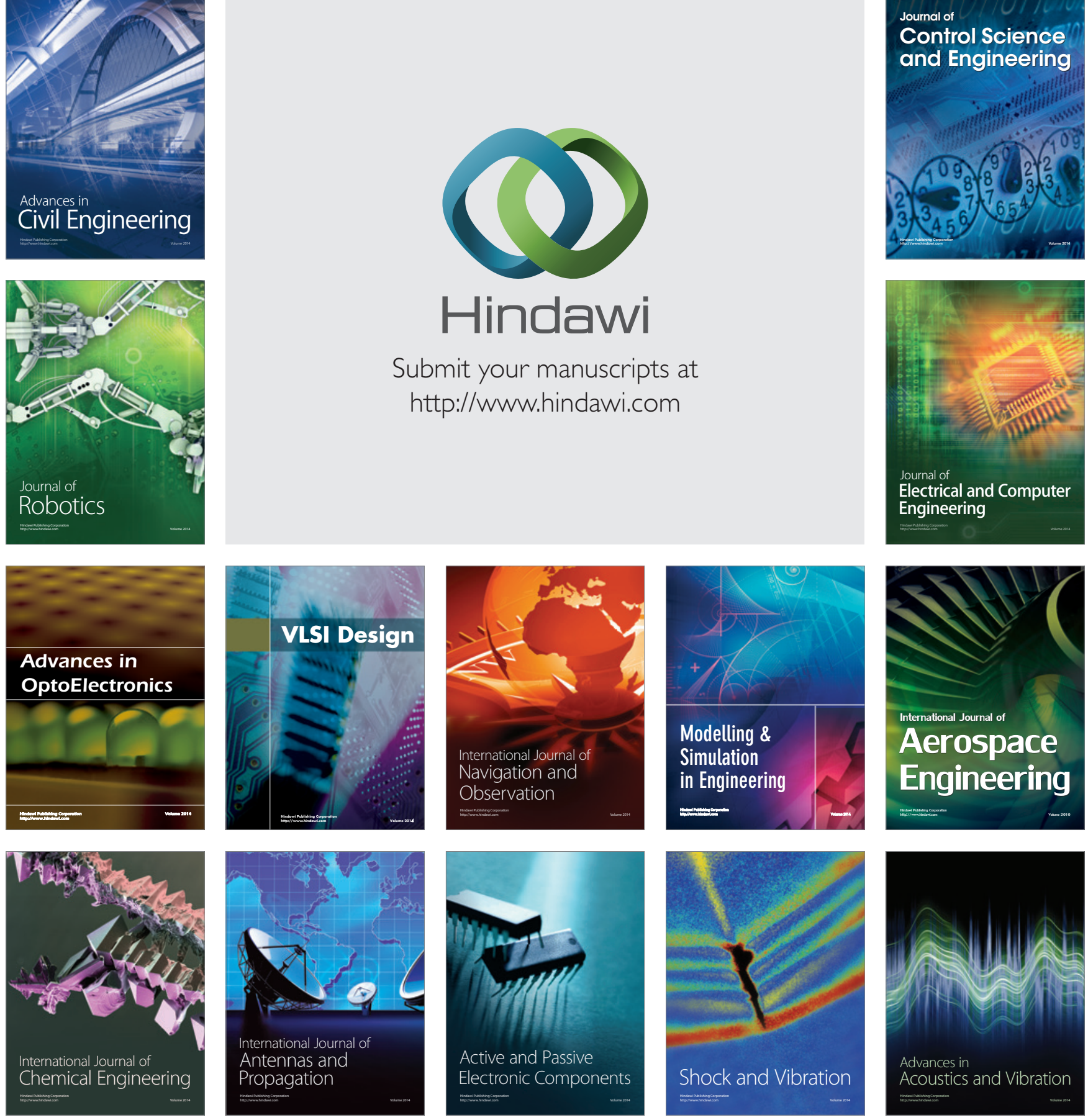